\title{
PRIMI RISULTATI SULLA LOCALIZZAZIONE DELLE PERTURBAZIONI ATMOSFERICHE DALLESAME DELLE ONDE MICROBARICHE
}

\author{
Ferruccio Mosetti
}

1. - Questo lavoro vuol essere l'introduzione ad un ciclo di ricerche miranti a definire la posizione ed il moto di perturbazioni atmo. sferiche quali cicloni, fronti, o nuclei di pressione, mediante il solo impiego di stazioni microlıarometriche; essendosi già precedentemente visto (1) the ogni tipo di perturbazione atmosferica genera onde microbariche caralleristiche. L'analisi dei microlıarogrammi dovreblue dunque poter permettere di inquadrare la situazione meteorica, entro un larghissimo ragrgio, di una zona interessante la stazione o il gruppo di stazioni di osservazione. È nostra idea che le onde microlariche si propaghino dalla zona perturlata alla stazione, direttamente, o indiretlamente per successive "altivazioni" delle masse d'aria attraversate. Tali onde sarehliero dunque di carattere progressiro, e, studiando la direzione e la distanza da cui provengono gli impulsi registrati (in lase alla differenza dei tempi di arrivo in tre stazioni e alla velociti di fase delle ondel, si potrelhe comodamente seguire sulla registrazione microharografica, il succedersi di eventi meteorici in zone anche molto lontane. Va notato che tale studio, cosi appena iniziato, è ostacolato da svariate difficolti alcune puramente tecniche, altre dovute al fatlo che fino a poco fa, nulla ci era noto sulla natura delle onde in questione. Anzitutto nessuna presupposizione potera esser fatta a priori riguardo la velocità delle onde microlsariche, perché tutti i pochi lavori eseguiti da Autori isolati sull'argomento della velocitì delle onde barometriche (anche a piccolo periorlo, per la loro scarsissima concordanza e per l'indole completamente diversa delle ricerche in cui apparivano, non ci poterano dare alcun affidamento, né erano snscettibili di generale applicazione. Dalle nostre misure al,hiamo notato una grande varialibitia della velociti, anche per onde aventi lo stesso periodo, per le più svariate cause, tanto che siamo ora del parere che la velocití, la cui conoscenza è essenziale per risolvere il nostro problema, sia da calcolarsi caso per caso. 
Anzitutto diremo che gia dalle prime osservazioni eseguite nell'estate di quest'anno con un gruppo di stazioni tripartite disposte a Trieste nelle localita: Osservatorio Geofisico, Roiano, Trebiciano, ai vertici cioè di un triangolo (fig. l) di lati dell'ordine di qualche chi-

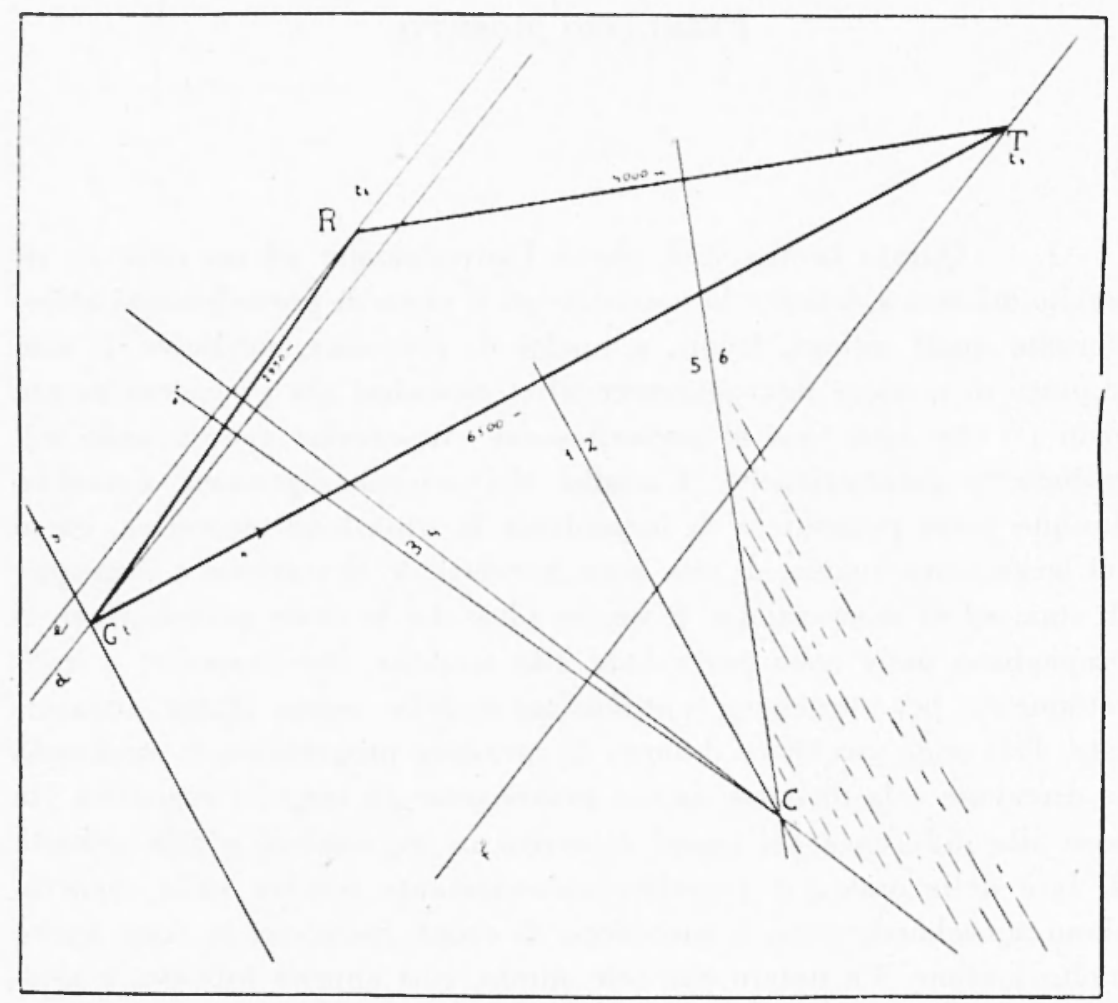

Fiz. 1

lometro, si intravvide la possibilita di risolvere il problema impostoci, e ciò per le seguenti ragioni: ogni impulso visibile in un microbarogramma era ritrovabile negli altri; di volta in volta gli sfasamenti risultavano diversi anche per gli stessi periodi; $i$ vari impulsi non venivano registrati simultaneamente nelle tre stazioni. Per ben poter discernere gli impulsi bisogna però tener conto che se i capillari dei microbarografi (-) non hanno sempre la medesima apertura, vi potrà esser sproporzione di ampiezza tra le varie registrazioni: se per esempio in una stazione il capillare è più chiuso che nelle altre, in questo strumento le onde di maggior periodo saranno esaltate e potranno 
mascherare gli impulsi di più breve periodo, che di solito hanno ampiezze minori; viceversa se il capillare è troppo aperto non si vedranno più grli impulsi superiori ad un certo periodo (-). Di tutto ciò lisognerà tenere accuratamente conto, anzi prossimamente la nostra ricerca si indirizzerì allo studlio di microbarogrammi di singrole bande di frequenza, ottenuti mediante opportuni filtri, per poter evitare al massimo la interferenza tra onde di varia natura e periodo, interferenza che può esser causa di errore sia nella valutazione del tempo di registrazione di un dato impulso, sia nella valutazione del periorlo di questo.

Inoltre, molte volte, negli strumenti di cui finora abljiamo fatto uso (sono quelli costruiti dal Bossolasco, modificati in modo da aver una registrazione ottica per eliminare l'incontrollabile attrito del penninol, che hanno uno scorrimento di $20 \mathrm{~mm}$ all'ora, gli sfasamenti sono spesso molto piccoli e difficilmente apprezzaloili con sicurezza. A tutte queste difficoltà stiamo ora cercando di ovviare, prima di intraprendere una ricerca definitiva sull'argomento, apportando notevoli migliorie agrli strumenti si da ottenere un tipo di microloarografo veramente rispondente allo scopo che oramai, per pratica e per esperienza, crediamo di ben conoscere.

Comunque sia, vogliamo ora dire come abhiamo cercato di risolvere il problema di ricavare, in hase alle due differenze di tempo delle registrazioni in tre stazioni, la velocita e la direzione di provenienza regrli impulsi. A prima vista, il problema potreble supporsi equale a quello che compare in Sismica, nella ricerca degli epicentri; senonché in Sismica nota rià da moltissimi anni la velociti delle onde, e costruite ottime tavole di dromocrone dalle quali si può ricavare la distanza in hase ai tempi di arrivo delle varie fasi, la ricerca odierna si riduce alla determinazione esatta (con i rati di più stazioni) della direzione cii provenienza, più che della distanza, (grià nota a priori con le dromocronel ed è questo un argomento molto studiato e per il quale esistono qià molti metodi. Nessuno di tali metodi poté però essere usato nella nostra ricerca, neanche quello fondamentale ed elementare delle iperlooli, che presuppone la conoscenza della velocita.

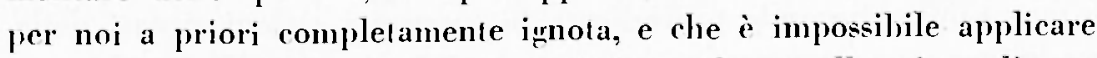
con sicurezza su distanze tanto grandi in confronto alle esigue dimensioni rlel triangolo usato.

Assolutamente impossibile poi, almeno per ora, è la costruzione di talıelle di dromocrone. Siamo ben lungri infatti dal poter ricavare. come in Sismica, la distanza, con i tempi di arrivo di eventuali fasi 
riflesse o rifratte, perché non sappiamo ancor nulla sulla eventuale possibilita di riflessione delle onde atmosferiche (longitudinali!) su qualche discontinuitì più o meno fissa (tropopausa?) e quindi sull'esistenza di onde riflesse di I, II, III ... ordine. Inoltre ancora, in Sismiea,

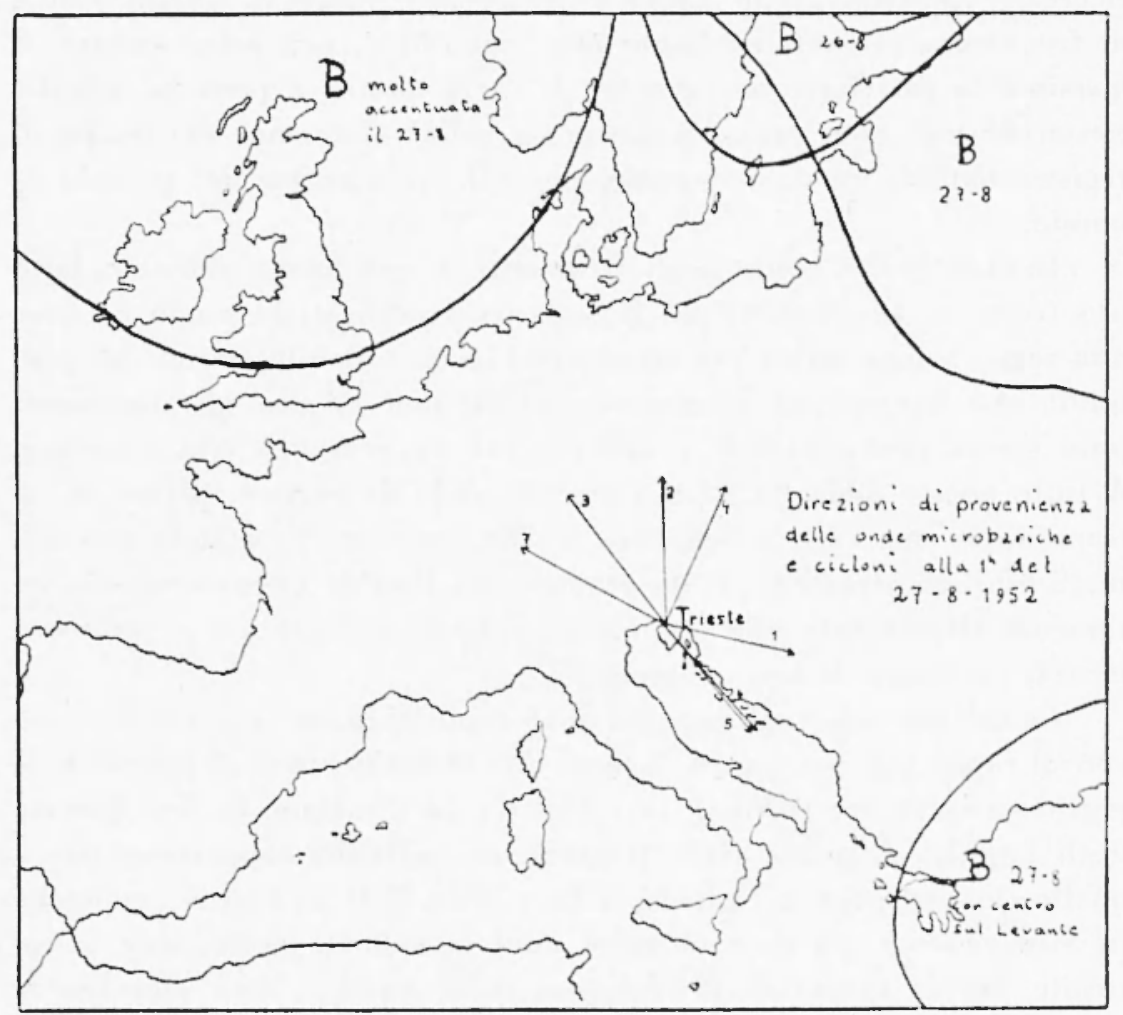

Fig. 2

onde che anche dopo anni provengono dalla riattivazione di una medesima zona ipocentrale, mantengono sempre eguale la loro velocita poiché rimangono inalterate le costanti del mezzo attraversato (per la relativa stabilità della crosta terrestre); mentre invece nel nostro caso è impossibile che onde provenienti da successivi cicloni situati nella medesima zona, mantengano invariata la velocità, per il fatto che con le stesse condizioni meteorologiche, variano continuamente temperatura, densità, umidita, pressione ecc. del mezzo attraversato. E poi ì chiaro che la velocità delle onde microbariche puio essere notevolmente influenzata dal movimento della sorgente (in Sismica, invece, l'ipocentro 


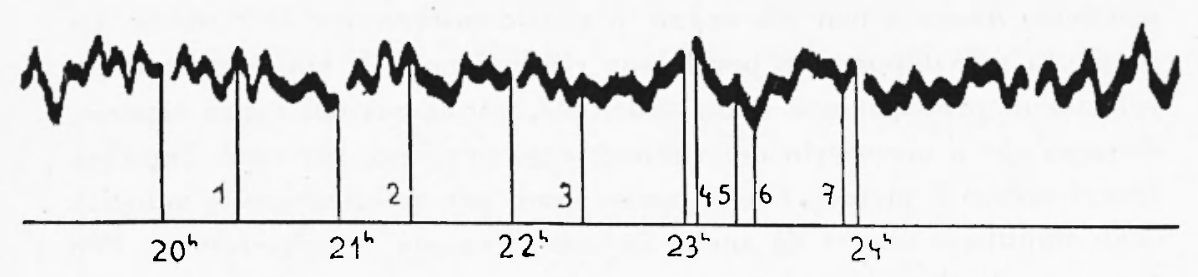

Geot. $27-8-52$

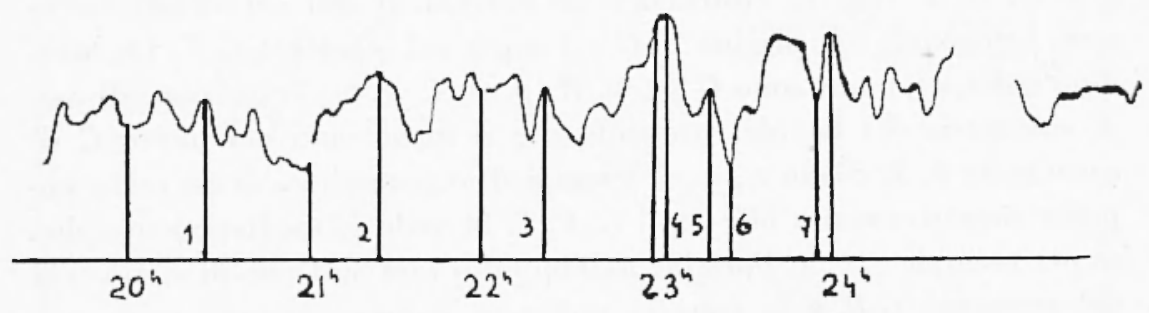

Roiano $27 \cdot 8-52$

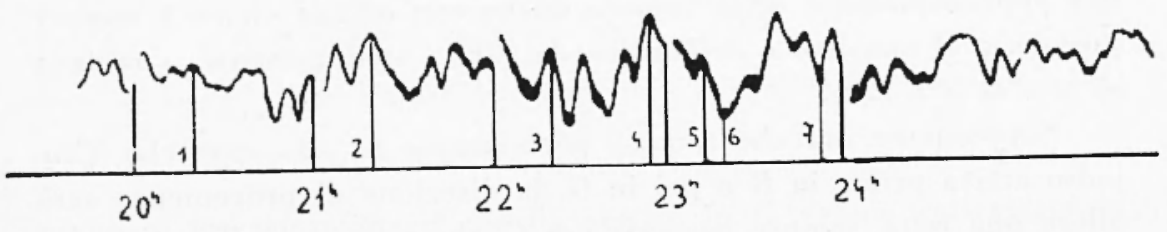

Treb. $27 \cdot 8 \cdot 52$

Fig. 3 
non si muove!) e dall'effetto di trascinamento dovuto al moto relativo delle masse d'aria attraversate. Ci troviamo insomma di fronte ad un problema nuovo e ben più arduo di quello interessante la Sismica. La premessa più importante per la sua risoluzione ì la conoscenza della velocità di propagazione delle onde, che, per le ragioni sopra esposte, diciamo che è necessario determinare cazo per caso, per ogni impulso. Descriveremo il metodo che abbiamo usato per determinare la velocita degli impulsi e che ci dà anche la loro direzione di provenienza. Per conoscere la distanza approssimata, usiamo per il momento semplici considerazioni sul periodo, che, aumenta chiaramente e, sembra, uniformemente con la distanza tra stazione e sorgente e varia poi, con la natura di questa.

2. - Il melodo usato è completamente analitico. Sia dato il triangolo $G, R, T$ (fig. 1), costruiamo un sistema di assi coordinati cartcsiani ortogonali con origine in $G$ e l'asse $x$ sul segmento G.T. Le coordinate dei tre punti sono $G(0,0), R(p, q), T(r, 0)$. Tracciamo gli assi di simmetria dei lati del triangolo che si incontrano nel punto $C$ di coordinate $h, k$. Siano $t_{1}, t_{0,}, t_{33}$ i tempi di registrazione di un certo impulso rispettivamente nei punti $G, R$. T. Si vede immediatamente che, se per esempio $t_{1}=t_{3}$ l'origine dell'impulso sarà sull'asse di simmetria del segmento $G \cdot R$ e la velocità sarà, con approssimazione tanto più buona quanto più lontana è l'origine (perché evidentemente il fronte d'onda non i sempre linearel, equale ai rapporto tra la distanza fra $G-R$ e la sua parallela condotta per $T$ e la differenza di tempo $t:-t_{1}$. Comunque abbiamo potuto vedere che l'errore che si commette in tale approssimazione della velocita anche con origini vicine $\dot{c}$ sempre piccolo, o al massimo è dell'ordine del $10 \%$. Analogamente si ragiona se $t_{1}=t_{3}$ o $t_{2}=t_{3 .}$.

Supponiamo ora che $t_{1} \neq t$. , per esempio $t_{1}>t .$, , cioi che l'impulso arriva prima in $R$ e poi in $G$, la direzione di provenienza sarà allora una rella, sempre passante per $C$, che non is piì l'asse di sim-

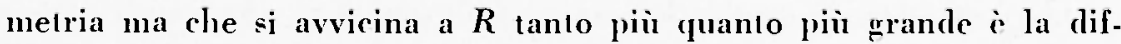
ferenza $t_{1}-t_{2}$. Naturalmente una tale determinazione della direzione di provenienza sarebbe laboriosa perché la posizione della rella che la rappresenta dipende non da una sola ma da tutte e tre le differenze dei tempi. Per ricavare almeno approssimativamente la direzione di provenienza si può ragionare anche nel seguente modo: sia per esempio $t_{1}>t_{.}>t_{3}$, vuol dire allora che l'impulso arriva prima in $T$ poi in $R$ e infine in $G$; il fallo che esso arrivi in $T$ prima che 
in $R$ vuol dire che la direzione di provenienza appartiene al semipiano 6 ( $f$ g. 1 ), questa retla appartiene anche al semipiano 4 , perché l'impulso arriva in $R$ prima che in $G$ e al semipiano 2 perché l'impulso arriva prima in $T$ che in $G$. In tal modo il settore che è co-

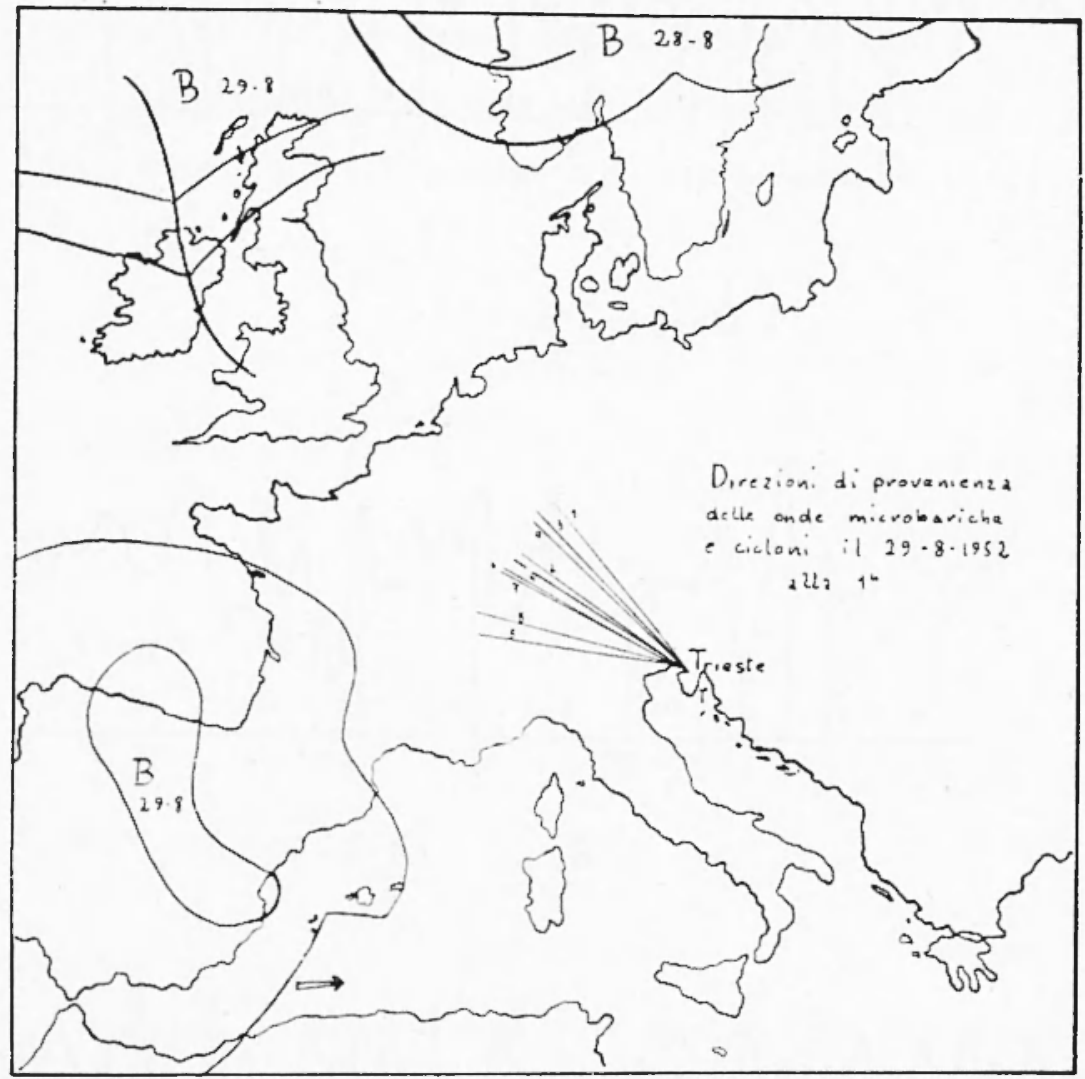

Fig. 4

mune ai tre semipiani, e che ha vertice in $C$, (zona tratteggiata in fig. 1), comprende la semiretta indicante la direzione di provenienza.

Da tutto questo si vede che, sempre, nel nostro metodo, la direzione di provenienza è rappresentata da una semiretta partente dal punto $C$. Sulla scorta di quanto abbiamo più sopra esposto pensiamo che, supponendo lineare (e si è dimostrato abhastanza ben approssimato) il fronte d'onda in un intorno del triangolo, si possono costruire tre rette parallele $d$, e ed $f$, passanti rispettivamente per i punti $G$, $R, T$, tali che la distanza tra $d$ ed $e$ sia proporzionale alla differenza 


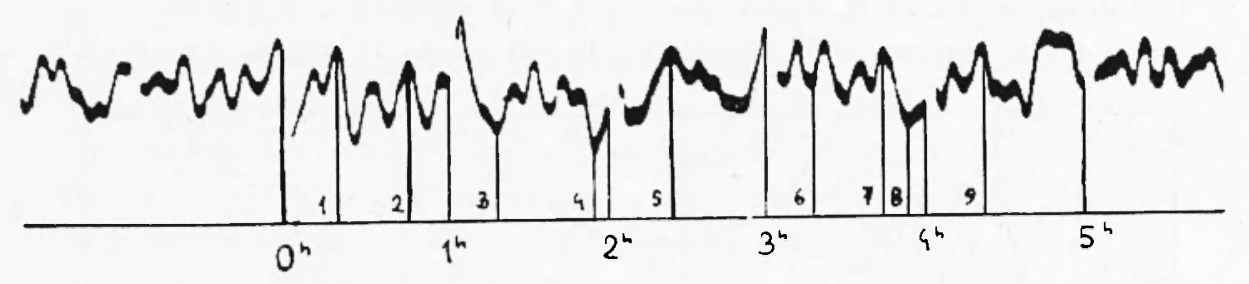

Geof. $29-8-52$

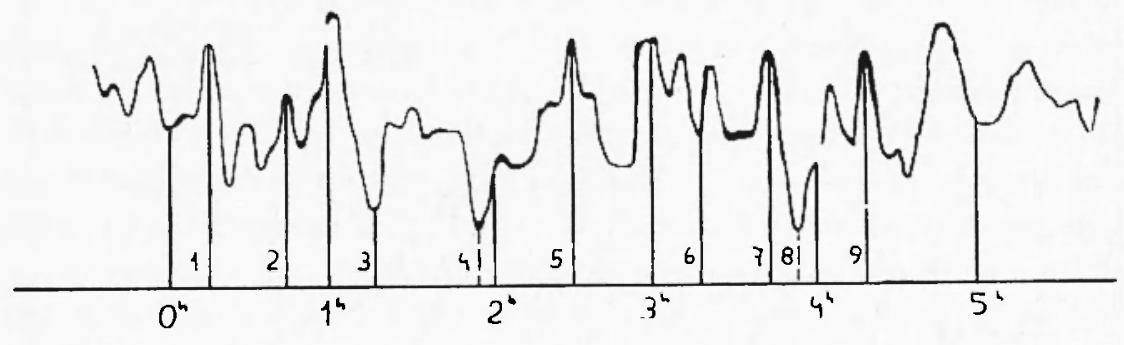

Roiano $29 \cdot 8 \cdot 52$

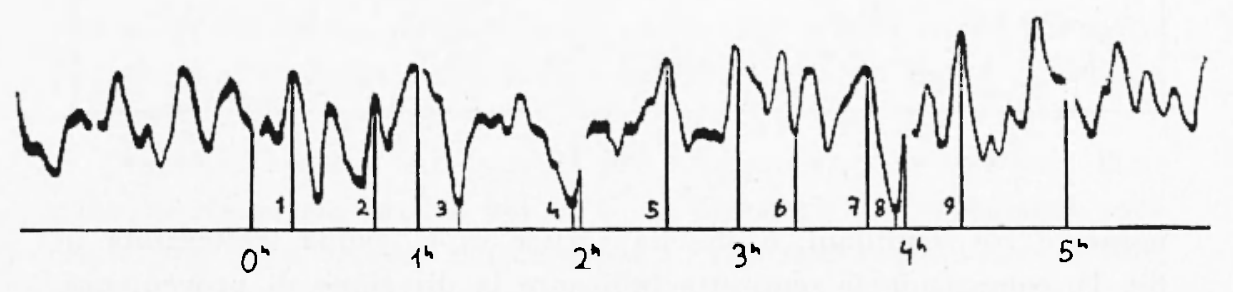

Treb. 29-8-52

Fig. 5 
$t_{2}-t_{1}$, e la distanza tra $d$ e $f$ sia proporzionale alla difierenza $t_{3}-t_{1}$ la perpendicolare a queste rette passante per $C$, sarà la direzione di provenienza cercata.

Sia

$a x+b y=0$ l'equazione della retta passata per $G(0,0)$;

$a(x-p)+b(y-q)=0$ l'equazione della retta per $R(p, q)$;

$a(x-p)+b y=0$ l'equazione della retta passante per $T(r, 0)$.

La distanza tra $d$ ed $e \dot{\mathrm{e}}$

$$
-\frac{a p+b q}{\sqrt{a^{2}+b^{2}}}
$$

Quella tra $d$ ed $f \dot{\mathrm{e}}$

$$
-\frac{a r}{\sqrt{a^{2}+b^{2}}}
$$

Occorrerà che sia

$$
\tau=\frac{t_{1}-t_{3}}{t_{1}-t_{9}}=\frac{a r}{a p+b_{l}}
$$

da cui

$$
\frac{a}{b}-\frac{q \tau}{r-p \tau}
$$

Il rapporto $-a / b$ è il coefficiente angolare delle rette parallele $d$, e ed $f$; quindi la direzione di provenienza avrà coefficiente angolare

$$
\frac{a}{b}=\frac{r-p \tau}{q \tau}
$$

l'equazione della retta rappresentante la direzione di provenienza (che passa per il punto $C(h, k)$ sarà dunque

$$
y=\frac{(k q-h p) \tau-h r}{q \tau}+\frac{r-p \tau}{q \tau} x
$$

Per ottenere la velocità basterà dividere per $\Delta t_{2}=t_{1}-t_{1}$ il valore assoluto della distanza tra $d$ ed $f$, o per $\Delta t_{1}=t_{1}-t_{2}$ il valore asso- 
luto della distanza tra $d$ ed $e$, ottenendo risultati necessariamente eguali.

\section{Infatti}

$\frac{a b+b q}{\sqrt{a^{2}+b^{2}}}=\frac{\frac{a}{b} p+q}{\sqrt[1]{\frac{a^{2}}{b^{2}}+1}}=\frac{\frac{p q \tau}{r-p \tau}+q}{\sqrt[1]{\frac{q^{2} \tau^{2}}{(r-p \tau)^{2}}+1}} \frac{q r}{\sqrt{\tau^{2}\left(q^{2}+p^{2}\right)+r^{2}-2 r p \tau}}$

e

$$
\frac{a r}{\sqrt{a^{2}+b^{2}}}=\frac{\frac{a}{b}:}{\sqrt{\frac{a^{2}}{b^{2}}+1}}-\frac{q r \tau}{\sqrt{\tau^{2}\left(q^{2}+p^{2}\right)+r^{2}-2 r p \tau}}
$$

dividendo la [1] per $\Delta t_{1}$ si ha

$$
v_{1}=\frac{q r}{\sqrt{\frac{\Delta t_{2}^{2}}{\Delta t_{1}^{2}}\left(q^{2}+p^{2}\right)+r^{2}-2 r p \frac{\Delta t_{0}}{\Delta t_{1}}}} \cdot \frac{1}{\Delta t_{1}}
$$

e dividendo la [2] per $\lrcorner t$

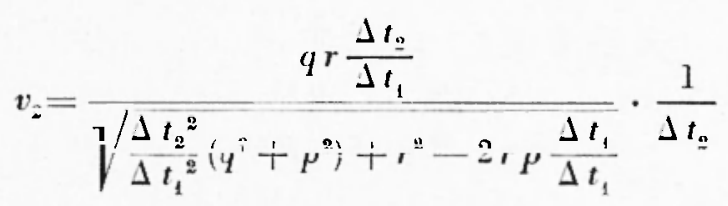

Queste due espressioni sono ovviamente eguali e si ottiene in definitiva per la velocita di fase la formula

$$
v=\frac{q r}{\sqrt{\left.\Delta t_{2}{ }^{2}\left(q^{2}+b^{2}\right)+\Delta t_{1}{ }^{2} r^{2}-2 r p \Delta t_{1}\right\lrcorner t_{2}}}
$$

Essendosi tale velocita ottenuta supponendo lineari i fronti d'onda, senza tener conto né del reale cammino del raggio nell'atmosfera. cammino che per ora si suppone superficiale né del moto della sorgente o del movimento cui sono soggette le successive zone d'aria attraversate, la denomineremo "velocita apparente " riservandoci in seguito, quando conosceremo meglio tutti i fenomeni cui è assoggettato 
il raggio d'onda nel suo cammino, di dare i valori della reale velo. cita di fase.

Per trovare il verso di un dato impulso hasterà guardare in quale dei tre punti esso arrivi per primo. Noti cosi direzione di provenienza

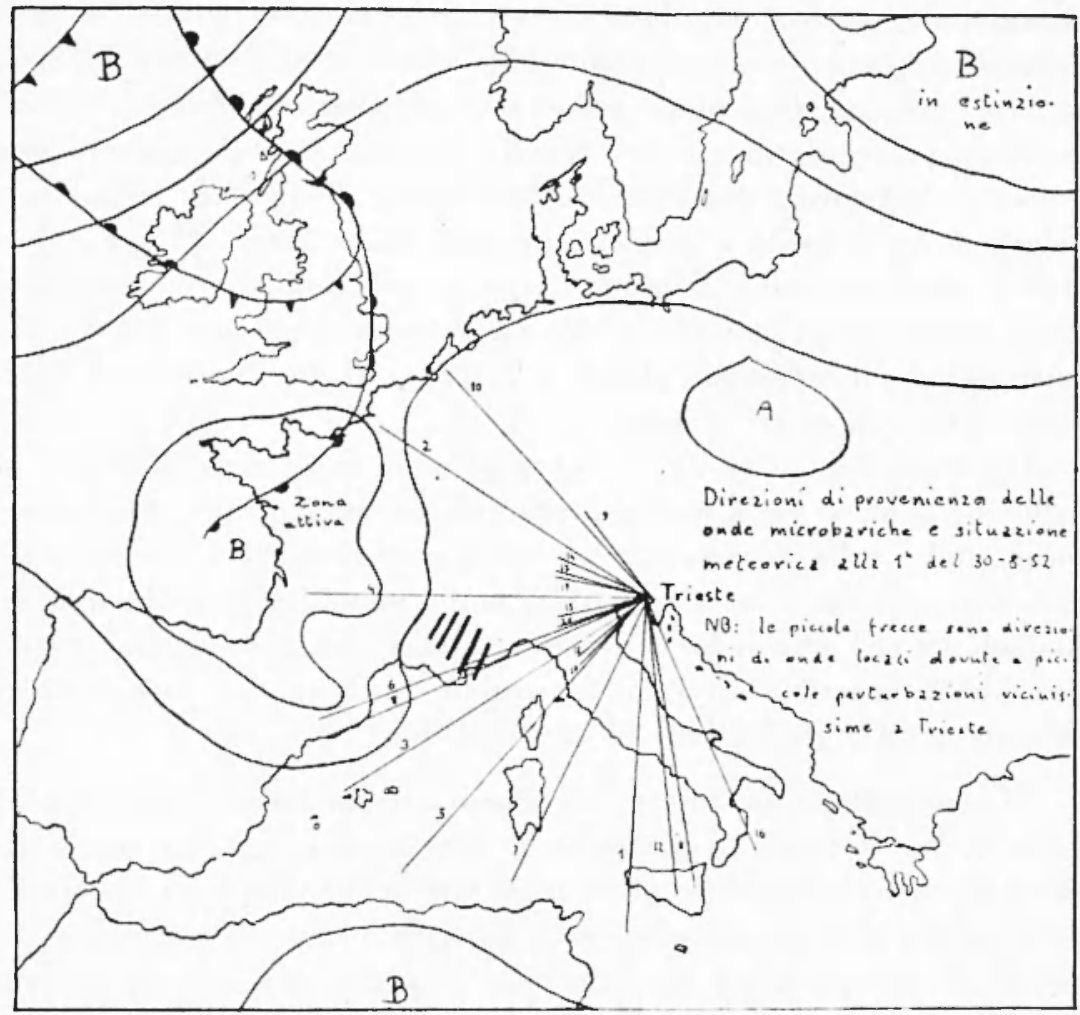

Fig. 6

e verso si applicano le semirette definite da queste due grandezze al punto $C$ e, misurando l'angolo che tali semirette formano in senso antiorario col meridiano passante per $C$ si ottiene l'azimul. Poiché le dimensioni del triangolo usato in questa ricerca risultano di solito molto piccole rispello alla distanza della perturbazione, non abbiamo potuto applicare alcun metodo atto a farcela conoscere con sicurezza. Però per poter sapere almeno grossolanamente la maggiore o minore lunghezza del cammino d'onda si può tener conto del fatto che $\left({ }^{1}\right)$ onde di periodo fino a $9,10 \mathrm{~min}$, di forma solitamente appuntata ed abbastanza ampie, sono di natura locale e provengono al massimo da una distanza di $100 \mathrm{~km}$. Onde di periodo sui $15,20 \mathrm{~min}$, di solito liscie 
ed abbastanza regolari, provengono da distanza dell'ordine di $1000 \mathrm{~km}$ c cosi via, quanto maggiore i il periodo e più regolari sono gli impulsi, tanto maggiore è la distanza della loro origine. Benché ci sia qualche relazione tra periodo e velocità per le onde proveniente dalla medesima perturhazione, onde di periodo eguale provenienti da perturbazioni pressoché equidistanti dalla stazione, ma situate in zone diverse, possono presentare velocità completamente diverse. Perciò avevamo precedentemente fatto notare che non si può assumere una velocità "standard ) per le onde microbariche, né definire alcuna correlazione tra velocita e periodo, in generale, a meno di non considerare comprese entro il limite di errore variazioni di velocita insite nella natura stessa del fenomeno. La velocita apparente delle onde microbariche è comunque piccola e varia tra alcune centinaia e qualche migliaio di metri al min!

Quando si analizza un microharogramma ci si trova di fronte al fat to che onde di varia natura e provenienza vengono registrate insieme e, molte volte, piccole e trascurabili perturbazioni vicine mascherano completamente le onde talvolta molto deboli, provenienti più da lontano. Sicché qua e là troveremo impulsi che provengono press'a poco dalla medesima direzione intercalati con altri di natura completamente diversa provenienti da tuttaltre zone.

3. - Mostreremo adesso con alcuni esempi come, calcolando il periodo, la velocità e la direzione di provenienza degli impulsi requistrati dal microbarografo si possa analizzare il microbarogramma traen. do fin d'ora utili informazioni sulla situazione meteorica locale e generale. Si vuol ancora notare che data la presenza e sovrapposizione di svariati sistemi di onde é difficile misurare il periodo di un impulso e che pertanto i valori indicati sono solo indicativi.

\begin{tabular}{|c|c|c|c|c|}
\hline $\begin{array}{l}\text { Caso del } 27-8 \cdot 1952 \\
\text { Intervallo di tempo } \\
\text { esaminato } \\
20^{\mathrm{h} .24 ! \mathrm{t}} \\
\text { fig. } 2 \text { e } 3\end{array}$ & \multicolumn{4}{|c|}{$\begin{array}{l}\text { Abbiamo scelto } 7 \text { impulsi nell'interval- } \\
\text { lo più interessante della registrazione. } \\
\text { Applicando le formule precedentemente } \\
\text { indicate si ricavarono i seguenti risultati }\end{array}$} \\
\hline n. imp. & $\mathbf{b} ; \mathbf{a}$ & azimut & $\begin{array}{l}\text { velocità } \\
\text { in } \mathrm{m} / \mathrm{min}\end{array}$ & $\begin{array}{l}\text { periodo } \\
\text { in min }\end{array}$ \\
\hline $\begin{array}{l}1 \\
2 \\
3 \\
4 \\
5 \\
6 \\
7\end{array}$ & $\begin{array}{l}-1,32 \\
+1,64 \\
-11,90 \\
+0,74 \\
-21,25 \\
-26,00 \\
-1,96\end{array}$ & $\begin{array}{r}-113^{\circ} \\
0^{\circ} \\
+\quad 35^{\circ} \\
-23^{\circ} \\
-142^{\circ} \\
-141^{\circ} \\
+59^{\circ}\end{array}$ & $\begin{array}{r}946 \\
.1600 \\
680 \\
570 \\
3680 \\
1165 \\
1690\end{array}$ & $\begin{array}{l}12 \\
20 \\
12 \\
24 \\
16 \\
12 \\
15\end{array}$ \\
\hline
\end{tabular}


(in figr. 3 per le localiti di Trebiciano l'inizio dell'ora è 5 min prima di quello segnato, causa ritardo del marcatempo).

Di questi impulsi il 2, 3 e il 7 provengono probabilmente da un forte ciclone centrato tra Scozia e Norvegia, il 4 da un ciclone sul Baltico, 1, 5 e 6 provengono da un ciclone sul Levante con centro rirca sull'Arabia.

\begin{tabular}{|c|c|c|c|c|}
\hline $\begin{array}{l}\text { Caso del } 29.8 \cdot 1952 \\
\text { Intervallo esaminato } \\
\text { 0h-5h } \\
\text { fig. } t \text { e } 5\end{array}$ & & & & \\
\hline 11. imp. & $b_{/}^{\prime} \mathbf{a}$ & azinut & velocità & periodo \\
\hline 1 & $-7,14$ & $+38^{\prime \prime}$ & 381 & 17 \\
\hline 2 & $-2,26$ & $+55^{\prime \prime}$ & 431 & 12 \\
\hline 3 & $-4,25$ & $+14^{\prime \prime}$ & 605 & 30 \\
\hline 4 & $-2,06$ & $+56^{\prime \prime}$ & 361 & 16 \\
\hline 5 & $-0,94$ & $+77^{\prime \prime}$ & 629 & 15 \\
\hline 6 & --1.89 & $+59^{n}$ & 365 & 12 \\
\hline 7 & $-1,76$ & $+60^{\circ}$ & 435 & 12 \\
\hline 8 & $-1,10$ & $+73^{0}$ & 459 & 19 \\
\hline 9 & $-4,85$ & +120 & 284 & 13 \\
\hline
\end{tabular}

(in fig. 5 l'ora del microbarogramma di Trebiciano è 6,4 min prima di quella segnata.

È interessante vedere come onde verisimilmente eguali per periodo e forma, (pur essendo più o meno distanti tra di loro nella registrazione, e "perturbate" da altre oscillazioni), quali le 2, 6, 7 e 4 provengano con valori di velocità molto vicini, dalla stessa direzione! Derivano probabilmente da un ciclone appena formato a $\mathbf{N}$ della Scozia. Le onde n. 1,3 e 9, di periodo più lungo, in media, delle altre potrebbero provenire vista la loro direzione da un vasto e profondo ciclone lontano estendentesi a NW di Trieste con centro presso la Norvegia. Gli impulsi 5 e 8 sarebbero dovuti ad una area ciclonica abbracciante il golfo di Guascogna e la Spagna. 


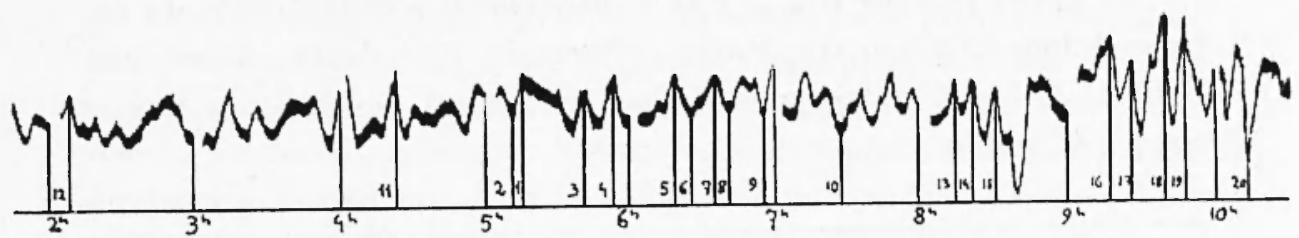

$$
\text { Geof } \quad 30.8 \cdot 52
$$

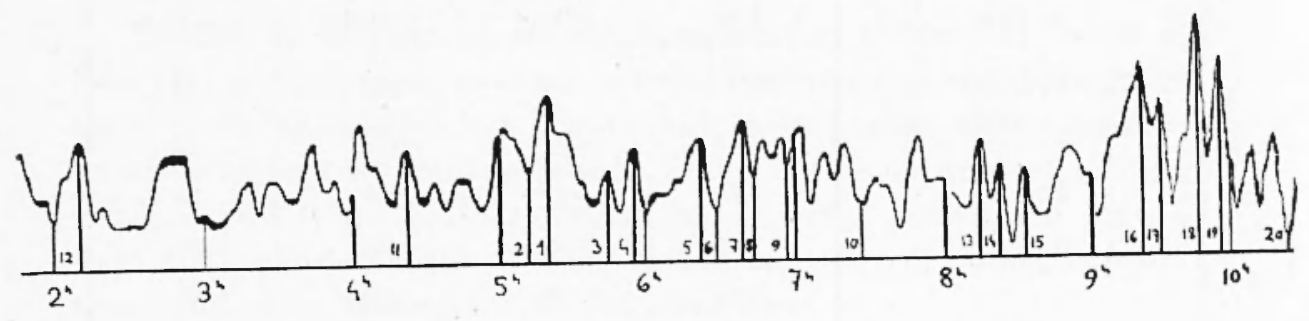

$$
\text { Roiano } 30 \cdot 8 \cdot 1952
$$

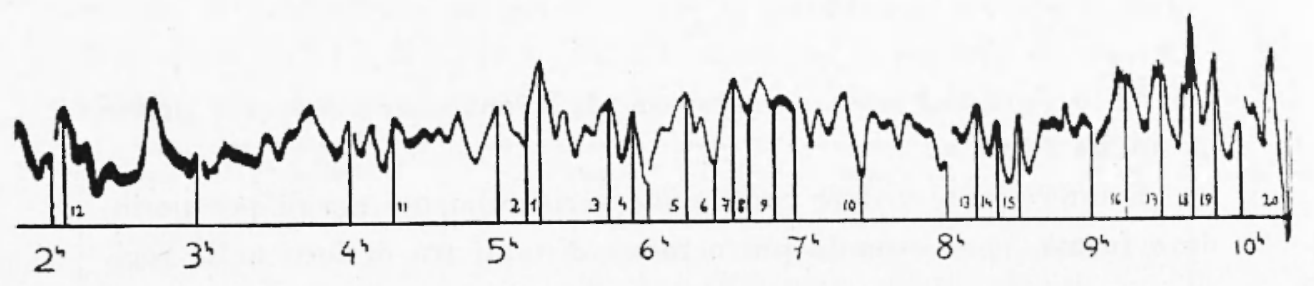

$$
\text { Treb. } 30 \cdot 8 \cdot 52
$$

Fig. 7 
(in fig. 7 l'ora del microbarogramma di Trebiciano cade $6,5 \mathrm{~min}$ prima di quella segnata).

\begin{tabular}{|c|c|c|c|c|}
\hline \multirow{2}{*}{$\begin{array}{l}\text { Caso del } 30-8-1952 \\
\text { Intervallo esaminato } \\
2^{\mathrm{h}}-{ }^{\mathrm{h}} \\
\text { fig. } 6 \text { e } 7 \\
\text { n. imp. }\end{array}$} & \multicolumn{4}{|c|}{$\begin{array}{l}\text { Rappresenta un esempio di analisi completa di un } \\
\text { tratto di microbarogramma dotato di onde di diverso } \\
\text { tipo e origine. }\end{array}$} \\
\hline & $b / a$ & azinut & velocità & periodo \\
\hline 1 & $+1,35$ & $+175^{\circ}$ & 625 & 30 \\
\hline 2 & $-2,15$ & $+55^{\circ}$ & 594 & 20 \\
\hline 3 & $\mathbf{0 , 0 0}$ & +1200 & 774 & 11 \\
\hline 4 & $-0,65$ & $+87^{\circ}$ & 735 & 11 \\
\hline 5 & $+0,25$ & $+135^{\circ}$ & 1720 & 20 \\
\hline 6 & $-0,21$ & $+108^{\circ}$ & 770 & 11 \\
\hline 7 & $+0,64$ & $+153^{\circ}$ & 1480 & 20 \\
\hline 8 & $-10,18$ & $+110^{\circ}$ & 1240 & 11 \\
\hline 9 & $-10,50$ & $+36^{\circ}$ & 267 & 8 \\
\hline 10 & $-6,06$ & $+40^{\circ}$ & 540 & 20 \\
\hline 11 & $+2,35$ & $+187^{\circ}$ & 1280 & 20 \\
\hline 12 & 2,02 & $+183^{\prime \prime}$ & 883 & 25 \\
\hline 13 & $-1,17$ & $+72^{\circ}$ & 989 & 8 \\
\hline 14 & $+1,61$ & $+62^{\circ}$ & 824 & 8 \\
\hline 15 & $-0,38$ & $+99^{\circ}$ & 1240 & 8 \\
\hline 16 & $-\nmid-3,08$ & $+191^{\circ}$ & 884 & 25 \\
\hline 17 & $-1,10$ & $+73^{\circ}$ & 746 & 9 \\
\hline 18 & $+0,16$ & $+133^{\circ}$ & 1100 & 9 \\
\hline 19 & $+0,23$ & $+129^{\prime \prime}$ & 885 & 9 \\
\hline 20 & -0.21 & $+108^{\circ}$ & 711 & 9 \\
\hline
\end{tabular}

Si nota sulle carte del tempo una zona perturbata in via di sfacelo (già analizzata col microbarogramma del giorno precedente) sull'Atlantico settentrionale da cui proverrebbero gli impulsi 2 e 10, di $20 \mathrm{~min}$ di periodo e velocità media di $567 \mathrm{~m} / \mathrm{min}$. Una zona perturbata sulla Francia e sul golfo di Guascogna, e in dipendenza di questa una zona tempestosa e molto attiva estendendosi fino alla Provenza e alle Alpi occidentali, provocherebbero gli impulsi 3, 4, 6 e 8, molto vicini per periodo e per velocità $\left(T=11 \mathrm{~min} ; v_{\mathrm{m}}=760 \mathrm{~m} / \mathrm{min}\right)$. Gli impulsi n. $1,5,7,11,12$ e 16 sono di origine molto lontana, provengono da qualche forte perturbazione africana non interessante la zona raffigurata dalle carte del tempo usate $(*)$, per esse velocità e periodo sono legate nel seguente modo:

$$
\begin{array}{r}
T=20^{\min } 25^{\mathrm{min}} 30^{\text {min }} \\
v_{\mathrm{m}}=1490884625 \mathrm{~m} / \mathrm{min}
\end{array}
$$

(*) Le carte del tempo consultate furono i Bollettini Quotidiani d'Informazioni del Servizio Meteorologico dell'Aereonautica di Roma. 
Gli impulsi 17, 18, 19 e 20 sono di origine vicinissima, dipendono da cause locali, (come si vede dal loro periodo e dalla loro forma) e son generati probahilmente da un temporale sull'Adriatico settentrionale o dall'avvicinarsi di una debole discontinuità non segnata sulle carte. Essi hanno periodo di 9 min circa e velocita media di 860 m/min. Cosi pure sono di origine locale gli impulsi 9, 13, 14, 15 di $8 \mathrm{~min}$ di periorlo e velocità media di $830 \mathrm{~m} / \mathrm{min}$.

4. - Sono questi appena i primi grossolani e sommari risultati che non possono essere definitivi ma che vogliono mostrare un metodo, una strada da seguire per potere col solo esame delle onde regristrate al microharografo, onde "dinamiche" di natura hen diversa dalle fluttuazioni (stazionarie) a lunghissimo periorlo, da altri sturliata, cercare di localizzare la causa che le senera e trarre cosi utili risultati pratici.

Istituto Nazionale di Geofisica -- Osserv. di Trieste - Ottobre 1952.

\section{RIASSUNTO}

Si prospetta la possibilità e il modo di loculizzare le perturba. zioni atmosferiche in base all'esame delle onde microbariche da que. ste generate. Si mostrano degli esempi da cui appare che la diresione di provenienza di particolari onde registrate dai microbarografi, corrisponde proprio alla posiziono dei cicloni rispetto al gruppo di stazioni registrutrici.

\section{SUMMARY}

It is considered possible to locate atmospheric perturbances by studying the microbaric-naves developped by the perturbances them. selves. From several examples it appears that the direction of particular waves recorded by microbarographs, correspond exactly to the position of cyclones in respect of the group of recording stations.

\section{BIBLIOGRAFIA}

(1) Mosettı F., Le onde microbariche. Rivista di Meteorologia Aereonatica III, 1952.

(-) Mosettı F., Teoria del microbarografo Allani, Ammali di Geofisica IV, 1951. 